\title{
Localization of the VP2 Protein of Canine Parvovirus Type 2 on the Baculovirus Envelop and Its Immunogenicity in a Mouse Model
}

\author{
Chih H. Tsai ${ }^{1}$, Jing Y. Wang ${ }^{2}$, Xin G. Xü ${ }^{2}$, De W. Tong ${ }^{2}$, Hsin Y. Lu ${ }^{3}$, Yi H. Chen ${ }^{3}$, Ming T. Chiou ${ }^{4}$, \\ Ching D. Chang ${ }^{4}$, Hung J. Liu ${ }^{3,5^{*}}$ \\ ${ }^{1}$ Graduate Institute of Vaccine Biotechnology, Pingtung, Taiwan \\ ${ }^{2}$ College of Veterinary Medicine, Northwest A\&F University, Yangling, China \\ ${ }^{3}$ Institute of Molecular Biology, National Chung Hsing University, Taichung, Taiwan \\ ${ }^{4}$ Department of Veterinary Medicine, National Pingtung University of Science and Technology, Neipu, Taiwan \\ ${ }^{5}$ Agricultural Biotechnology Center, National Chung Hsing University, Taichung, Taiwan

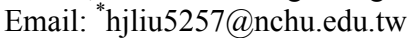

Received September 4, 2012; revised October 12, 2012; accepted October 25, 2012

\begin{abstract}
In this study, the full-length VP2 gene of canine parvovirus type 2 (CPV-2) was cloned into the pBacSC vector which possesses baculovirus transmembrane domain (gp64 TM) gene, baculovirus cytoplasmic domain (gp64 CTD) gene, and green florescence protein (GFP) gene. Baculovirus gp64 TM and gp64 CTD in the pBacSC vector were designed to display heterologous proteins on the baculovirus envelope. After cloning the VP2 gene of CPV-2 into pBacSC vector, the recombinant plasmid pBacSC-VP2 was transformed into E. coli DH10Bac competent cells to form recombinant bacmid DNA. One recombinant baculovirus BacSC-VP2 that expresses the VP2 protein of CPV-2 was obtained. Confocal microscopy and immunogold electron microscopy were used to verify whether VP2 expressing on baculovirus envelope or cell membrane. Immunization of BALB/c mice with recombinant baculovirus BacSC-VP2, demonstrated that serum from the BacSC-VP2 treated models had higher levels of virus neutralization titers than the control groups. The results show that the recombinant baculovirus BacSC-VP2 can induce a strong immune response in a mouse model, suggesting that the pseudotyped baculovirus BacSC-VP2 can serve as a potential vaccine against CPV infections.
\end{abstract}

Keywords: Canine Parvovirus Type 2; VP2 Protein; Baculovirus gp64 TM and CTD; Subunit Vaccine

\section{Introduction}

Among carnivore parvoviruses, feline pan leukopenia virus (FPLV) has been known since the 1920s, whereas canine parvovirus (CPV) only emerged as a dog pathogen in the late 1970s [1]. CPV emerged in 1978 worldwide and was termed CPV-2 to distinguish it from CPV-1 (minute virus of canines [2]. CPV-2 is the etiological agent of an epizootic severe gastroenteritis of dogs characterized by depression, loss of appetite, vomiting, diarrhea and leucopenia [3-5]. CPV-2 contains a single-stranded and negative-sense DNA genome of about $5.2 \mathrm{~kb}$ that encodes two structural proteins (VP1 and VP2) and two nonstructural proteins (NS1 and NS2). The major capsid protein VP2 was reported to play an important role in virus pathogenicity and the host immune response [6]. Since there are reports that only a few amino acid substitutions in VP2 are responsible for its antigenic properties [7], its gene sequence has been re-

${ }^{*}$ Corresponding author. garded as the most useful marker for revealing the genetic relationships of CPV-2, as well as serving as an important target for antigenic and epidemiologic studies of the virus $[8,9]$. The original CPV-2 evolved continuously and was completely replaced by three new types, CPV-2a, CPV-2b, and CPV-2C [10].

In this study, we have succeeded in constructing a novel baculovirus-based system to display VP2 protein of CPV-2 154 strain on the baculovirus envelope or cell membrane. Our results show that the new development of pseudotyped baculovirus BacSC-VP2 can induce robust immune response in a mouse model, indicating that the pseudotyped baculovirus BacSC-VP2 can serve as a potential vaccine against $\mathrm{CPV}$ infections.

\section{Materials and Methods}

\subsection{Cells and Viruses}

The Spodoptera frugiperda (Sf-9) cell lines were grown as monolayers in TNM-FH medium (Sigma, St. Louis, 
MO, USA) supplemented with $10 \%$ heat-inactivated fetal bovine serum (Invitrogen Co., Carlsbad, USA), 100 U/ml of penicillin and $100 \mu \mathrm{g} / \mathrm{ml}$ of streptomycin. Recombinant viruses were propagated and tittered in Sf-9 cells. Proteins were also expressed in Sf-9 cells.

\subsection{Preparation of Recombinant Bacmid DNA}

The VP2-encoding gene of CPV-2 154 strain was amplified by using primers CPV-VP2-1 and CPV-VP2-2. CPV-2 VP2-targeted primers for amplification of extracted DNA were designed. The primers used to amplify the VP2-encoding gene were shown in Table $\mathbf{1}$. The amplified product was cloned into the corresponding sites in the plasmid $\mathrm{pBacSC}$ vector developed by our laboratory [11]. The resultant construct pBacSC-VP2 was verified by enzyme digestion and DNA sequencing. Competent DH10Bac E. coli cells were then transformed with the recombinant plasmid pBacSC-VP2 and the vector $(\mathrm{pBacSC})$ alone. The vector transformats were used as a negative control. After selecting colonies through two rounds of blue/white selection, recombinant bacmids were isolated from white colonies using the procedure provided by Invitrogen Co. The recombinant clones were then checked for the presence of the insert by PCR using primers CPV-VP2-1 and CPV-VP2-2. The positive colonies were cultured to isolate the bacmid DNA. The recombinant baculoviral DNA was named as pBacSC-VP2.

\subsection{Transfection and Construction of Recombinant Baculoviruses}

All procedures for the production of viral particles were performed according to the manufacturer's manual (Invitrogen). Briefly Sf-9 was allowed to attach for $1 \mathrm{~h}$. After attachment, four micrograms of recombinant bacmid DNA BacSC-VP2 in $10 \mu \mathrm{l}$ lipofectaine 2000 (Invitrogen) were transfected into $80 \%$ confluent Sf-9 cells in 6-well plates. Transfected cells were incubated for $5 \mathrm{~h}$ at $27^{\circ} \mathrm{C}$ and the transfection medium was replaced with fresh medium. After incubation for $72 \mathrm{~h}$ at $27^{\circ} \mathrm{C}$, the recombinant viruses were chosen based on GFP expression and purified by three rounds of plaque isolations. Individual recombinant viruses were tittered by plaque assay and high titer stocks were used for infecting the cells. The recombinant baculovirus was named as BacSC-VP2.

\subsection{Sodium Dodecyl Sulfate-Polyacrylamide Gel Electrophoresis (SDS-PAGE) and Western Blot Analysis}

The infected cell lysates were subjected to $10 \%$ SDSPAGE and transferred to nitrocellulose membranes. Two primary antibodies (anti-His ${ }_{6}$ monoclonal antibody 1: 3000 dilution and anti-CPV-2 polyclonal antibody 1:1000 dilution) were used to detect CPV VP2 protein in the
Western blot. The secondary antibody was goat antimouse IgG conjugated to HRP (1:5000 dilution; KPL, Gaithersburg, Maryland, USA). The protein bands were visualized by the ECL chemiluminescence system on Hyper-Max films, as recommended by the manufacturer (Amersham Pharmacia Biotech, Hong Kong).

\subsection{Confocal Microscopy}

The Sf-9 cells were cultured on sterile cover slips (placed in six-well plates) and infected at an MOI of 10. Two days after infection, the cells were fixed by methanol/acetone $(1: 1)$ for 5 minutes at $-20^{\circ} \mathrm{C}$, rinsed with PBS, and blocked with $2 \%$ bovine serum albumin for 30 minutes at $37^{\circ} \mathrm{C}$. The cells were then incubated with the primary antibody (anti-CPV-2 polyclonal antibody, 1:1000 dilution and anti-His ${ }_{6}$ monoclonal antibody 1:3000 dilution) for 1 hour at $37^{\circ} \mathrm{C}$, followed by three PBS washes. The cells were then incubated with the secondary antibody (FITC-conjugated goat anti-mouse monoclonal antibody 1:200 dilution; KPL) for $1 \mathrm{~h}$ at $37^{\circ} \mathrm{C}$, followed by three PBS washes. The negative controls were treated the same way. Protein localization was visualized using a confocal microscope (LSM 510, Zeiss, Thornwood, USA).

\subsection{Immunogold Electron Microscopy}

To prepare the purified virus particles for immunogold electron microscopy, the recombinant baculovirus BacSC-VP2 was produced by infecting Sf-9 cells at an MOI of 0.1 and harvested 4 days after infection. The virus supernatant was purified by two rounds of sucrose gradient ultracentrifugation following standard protocols. The immunogold electron microscopy was performed as described previously [11-13] with some modifications. The carbon-coated grids were floated on $10 \mu \mathrm{l}$ purified baculovirus solution for 30 minutes, blocked, washed with PBS three times for 5 minutes, and then exposed to anti-His $_{6}$ monoclonal antibody as the primary antibody (1:100 dilutions) for 30 minutes. After two PBS washes, the grids were exposed to anti-mouse $\operatorname{IgG}$ conjugated with 5-nm gold particles (1:50 dilution) (Sigma, St. Louis, USA) for 30 minutes. After three more PBS washes, the grids were stained with $2 \%$ phosphotungstic acid (Sigma) and examined under a transmission electron microscope (H-7500, Hitachi, Tokyo, Japan).

\subsection{Immunization and Measurement of CPV-2 Titer}

Four groups of mice, five females in each group, were immunized intraperitioneally with $3 \times 10^{8}$ pfu of purified BacSC-VP2. As negative controls, five mice were injected with purified $\mathrm{BacSC}\left(3 \times 10^{8} \mathrm{pfu}\right)$ or phosphate 
buffered saline (PBS) and five mice were injected with CPV-2 vaccine (1 dose) as a positive control. Each mouse received one booster shot 2 weeks following initial injection. The blood samples for ELISA were collected 6 weeks after initial injection. The ELISA kit used in this study was from Ingenasa Co. (Madrid, Spain). ELISA titers were converted into serum neutralization (SN) titers by using the formula provided by manufacture.

\subsection{Statistical Analysis}

All data were analyzed using independent sample t-test and are expressed as averages of three independent experiments. $P$ value of less than 0.05 was considered significant.

\section{Results}

\subsection{Construction of the Recombinant Bacmid}

Recombinant baculoviral DNA harboring the VP2-encoding gene of CPV-2 154 strain was prepared using the pBacSC vector containing baculovirus gp64 SS, His 6 , multiple cloning sites localized between $\mathrm{His}_{6}$ and baculovirus gp64 CTD, baculovirus gp64 CTD, baculovirus gp64 TM, and enhanced green fluorescent protein (EGFP). Figure 1 shows the strategy for cloning of the $\mathrm{VP} 2$-encoding gene of CPV-2 in the pBacSC vector.

\subsection{Confirmation of VP2 of CPV-2 Expression in Insect Cells}

To verify the expression of $\mathrm{His}_{6}$-tagged VP2 protein in insect cells, Sf-9 cells were infected by BacSC-VP2 recombinant baculovirus at a multiplicity of infection (MOI) of 10, and harvested 3 days after infection. There was no target protein expression detected by anti-His ${ }_{6}$ or anti-CPV-2 antibodies in the negative controls (BacSC \&

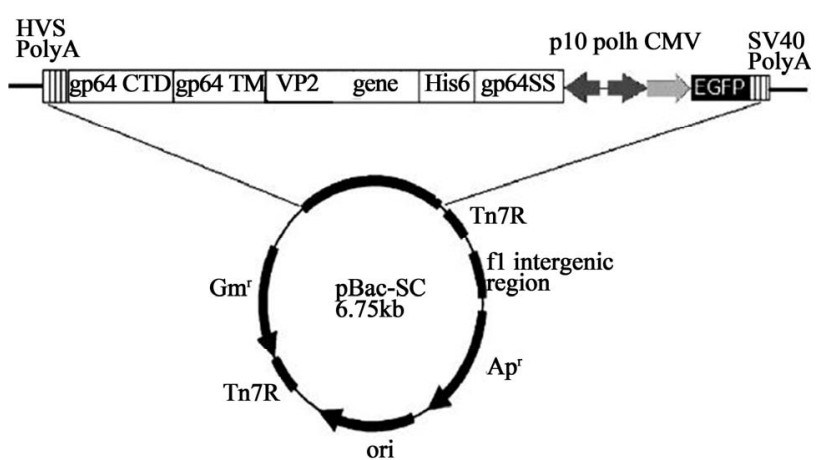

Figure 1. Illustration of the constructed recombinant baculovirus vector. Using CPV 154 genome as a template, the VP2-encoging gene of CPV was amplified by primers CPV-VP2-1 and CPV-VP2-2. The VP2 gene of CPV was cloned into the pBacSC vector. The construct was named as pBacSC-VP2.
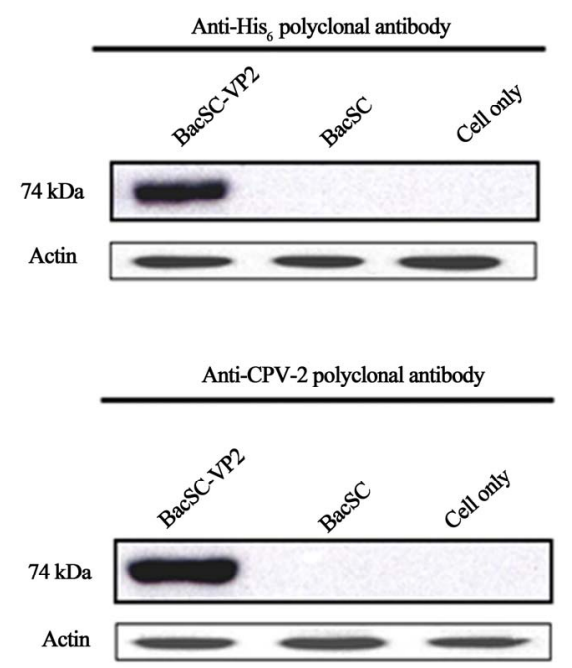

Figure 2. Confirmation of the expression of CPV-2 VP2 protein in Sf-9 cells by Western blot assay. The cells were infected with BacSC-VP2 baculovirus at an MOI of 10, harvested 3 days post-infection. The harvested proteins were subjected to Western blotting assay using anti-His monoclonal and anti-CPV-2 polyclonal antibodies. Blots are representative for three experiments.

Sf-9 cells) (Figure 2). In contrast, the VP2 protein (74 $\mathrm{kDa}$ ) expressed by BacSC-VP2 was detected by antiCPV-2 polyclonal antibodies (Figure 2).

We attempted to further examine whether the $\mathrm{His}_{6}{ }^{-}$ tagged VP2 protein was properly translocated to the cell surface, the cells were cultured on sterile cover slips, infected with baculovirus at an MOI of 10, and subjected to immunofluorescence labeling/ confocal microscopy 2 days after infection. There were no proteins detectable by anti-His $_{6}$ or anti-CPV-2 antibodies in the negative controls (BacSC) (Figure 3). In contrast, our confocal microscopy results revealed that VP2 was directed to the plasma membrane of the infected Sf-9 cells (Figure 3).

\subsection{Sodium Dodecylsufate-Polyacrylamide Gel Electrophoresis (SDS-PAGE) and Western Blot of Purified Recombinant Baculovirus BacSC-VP2}

The incorporation of $\mathrm{His}_{6}$-tagged VP2 into baculoviruses was probed using the anti-His 6 and anti-CPV-2 antibodies, respectively. In the present study, there was no target protein expression in the negative control BacSC (Figure 4). In contrast, the VP2 protein expressed by BacSC-VP2 was detected by above antibodies.

\subsection{Evaluation of Display of CPV-2 VP2 Protein on the Baculoviral Envelope}

To provide more concrete evidence for VP2 displayed on the baculoviral envelope, recombinant baculoviruses BacSC-VP2 was concentrated by sucrose cushion gradient 

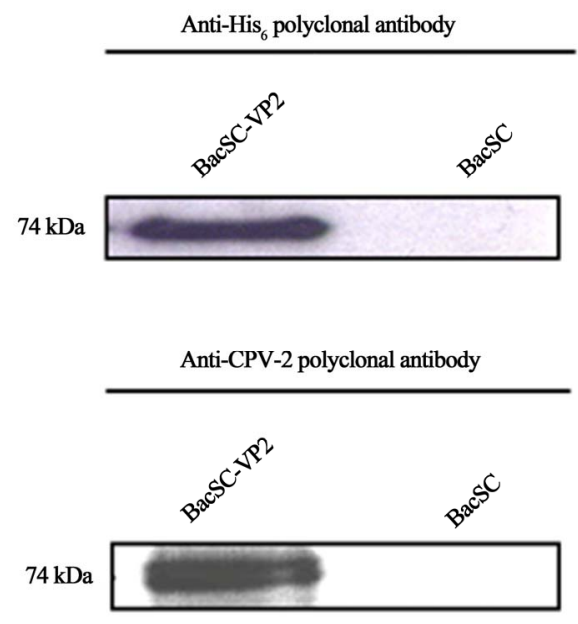

Figure 3. The CPV-2 VP2 protein expression in concentrated recombinant baculoviruses confirmed by Western blot assay. The cells were infected with BacSC-VP2 baculovirus at an MOI of 10, and harvested 3 days postinfection and subjected to Western blot assay using anti-His monoclonal and anti-CPV-2 polyclonal antibodies.

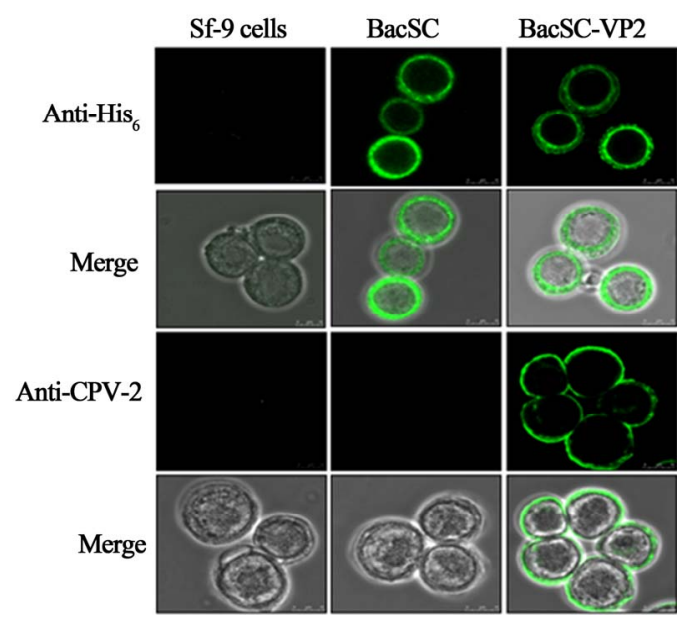

Figure 4. Confirmation of expression of VP2 protein by confocal microscopy. The cells were cultured on sterile cover slips, infected with BacSC-VP2 baculovirus at a MOI of 10, subjected to immunofluorescence staining and observed with confocal microscopy 2 days postinfection. The figure shows the detection of the BacSC-VP2 recombinant baculovirus expressed VP2 protein by anti-His monoclonal and anti-CPV-2 polyclonal antibodies. The protein localized to the plasma membrane, thus demonstrating the anchoring of VP2 on the surface of Sf-9 cells.

ultracentrifugation and subjected to immunogold electron microscopy with anti-His ${ }_{6}$ monoclonal antibody as a primary antibody and anti-mouse IgG secondary antibody conjugated with 5-nm gold particles. As expected, we observed the distribution of gold particles only on the surface of BacSC-VP2 which confirms the incorporation of His $_{6}$-tagged VP2 on the baculovirus envelope (Figure 5).

Whether the choice of baculovirus gp64 CTD influ-

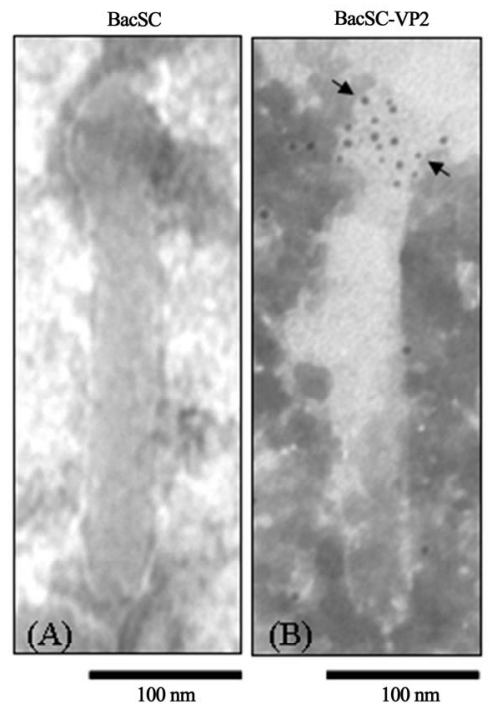

Figure 5. Immunogold electron micrographs of purified recombinant baculovirus. Using anti-His ${ }_{6}$ monoclonal antibody as the primary antibody and anti-mouse IgG conjugated with 5-nm gold particles as the secondary antibody. Arrows indicate the VP2 protein of CPV-2 displayed on the viral envelope.

enced the amount of CPV-2 VP2 incorporated into baculovirus, SDS-PAGE and Western blot assay as well as quantified by scanning densitometry were used to examine the expression level of gp64 and CPV-2 VP2 (Figure 6). The amounts of incorporated His6-tagged VP2 and gp64 were normalized on the basis of equal amounts of the major capsid protein VP39 of baculovirus. In this study, the negative control (BacSC) has similar efficiency of incorporation of gp64, as compared to BacSC-VP2 ( $\mathrm{P}>0.05)$ (Figure 6). Furthermore, compared with our previous constructed recombinant baculovirus BacHA64 [12], the incorporation of VP2 of CPV-2 and HA of avian influenza virus (AIV) and their display on the baculoviral envelope exhibited no difference $(\mathrm{P}>0.05)$ (Figure 7), suggesting that protein structure did not affect the efficiency of incorporation and virus morphology.

\subsection{Immune Response of CPV-2 VP2-Pseudotyped Baculoviruses}

To further investigate whether CPV-2 VP2 protein displayed on the baculoviral envelope could serve as an immunogen in vivo, one group of five female $\mathrm{BALB} / \mathrm{c}$ mice were immunized intraperitoneally with $3 \times 10^{8} \mathrm{pfu}$ of purified BacSC-VP2. Ten mice were injected with purified BacSC $\left(3 \times 10^{8} \mathrm{pfu}\right)$ and PBS, respectively. As shown in Figure 8. The $\mathrm{OD}_{450}$ value was about 0.2 in the mice immunized with the BacSC or PBS. Intriguingly, $\mathrm{OD}_{450}$ value went up to 3.3 in mice immunized with 

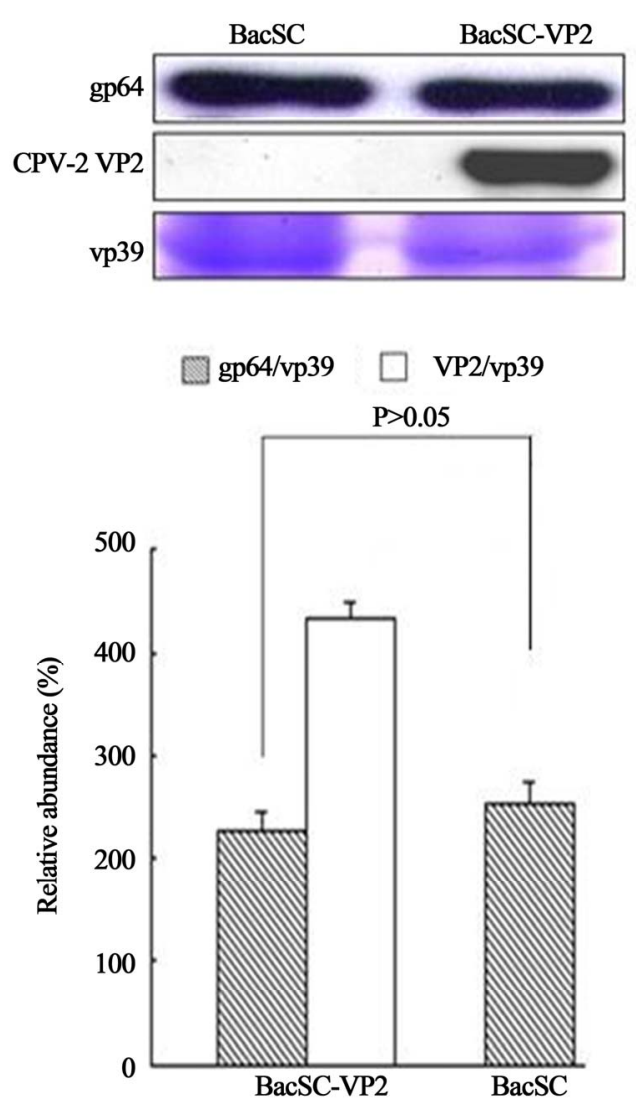

Figure 6. SDS-PAGE and Western blot of purified recombinant baculoviruses BacSC-VP2. The amount of virus was calibrated to the amount of $\mathrm{vp39}$. The band intensities for VP2 and gp64 on the Western blot, as well as the band intensities for vp39 on the SDS-PAGE, were scanned and analyzed using Scion Image Shareware. The data represent the mean \pm SD of three independent experiments.

BacSC-VP2 and to about 1.8 in mice immunized with $\mathrm{CPV}$ vaccine. The results suggest that the pseudotyped baculoviruses BacSC-VP2 successfully elicits immune response. Antibodies against the VP2 protein of CPV-2 expressed on the baculoviral virus particles, showed higher levels of virus neutralization activities. Compared to negative controls (BacSC and PBS), there were significant differences $(\mathrm{P}<0.05)$. As shown in Table 2, no detectable virus neutralization titers of VP2 were present in the mice immunized with the negative controls (BacSC and PBS). The virus neutralization titers of the displayed BacSC-VP2 were much higher than those of the positive control ( $\mathrm{CPV}$ vaccine) $(\mathrm{P}<0.05)$ (Table 2$)$.

\section{Discussion}

CPV-2 is responsible for acute hemorrhagic enteritis and fatal myocarditis in domestic dogs as well as some wild carnivore species. At present, the original CPV-2 is not circulating in dog populations, although it is still present in vaccine formulations $[14,15]$, whereas the variants
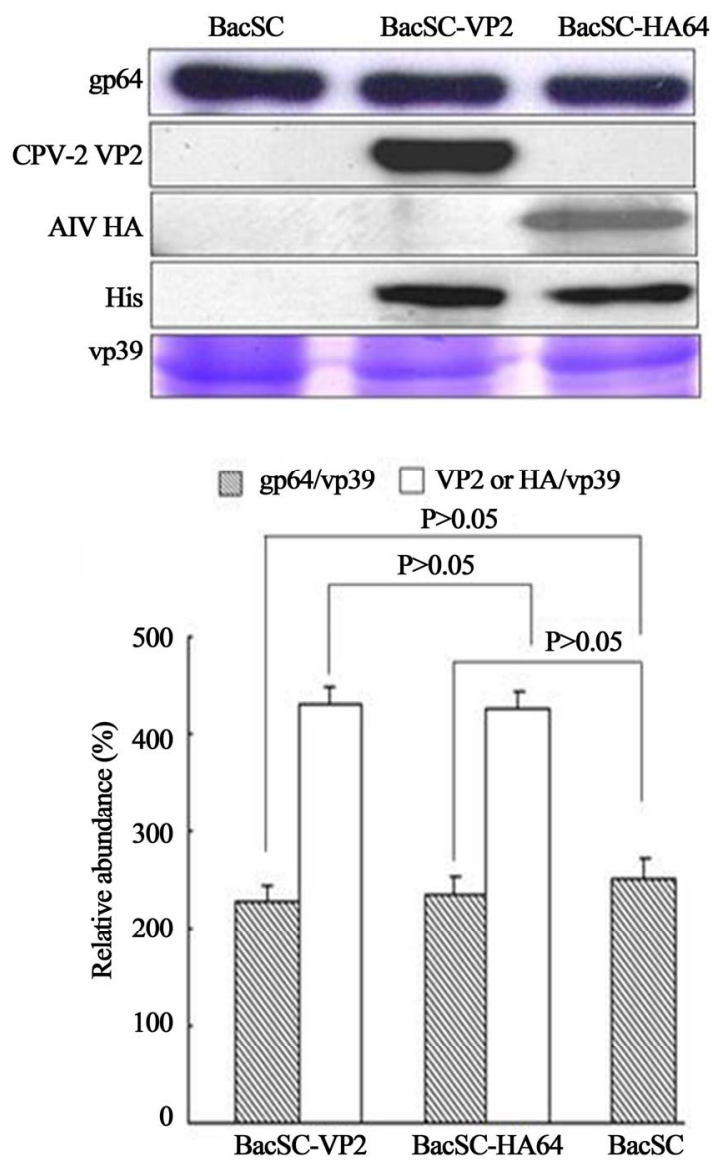

Figure 7. SDS-PAGE and Western blot of purified recombinant baculoviruses (BacSC-VP2 and BacSC-HA64). The amount of virus was calibrated to the amount of vp39. The band intensities for VP2, HA and gp64 on the Western blot, as well as the band intensities for vp39 on the SDS-PAGE, were scanned and analyzed using Scion Image Shareware. The data represent the mean \pm SD of three independent experiments.

Table 1. CPV primers used in this study.

\begin{tabular}{cc}
\hline CPV-VP2-1 & 5'-CAATCTCGAGccaatgagtgatggagcagttcaacc-3' \\
\hline CPV-VP2-2 & 5'-AAGTGAATTCatataatttctaggtgctagttgag-3' \\
\hline
\end{tabular}

Forward primer restriction enzyme: Xho I, Reverse primer restriction enzyme: EcoR I.

Table 2. Virus neutralization (VN) titers in BALB/c mice immunized with various immunogens.

\begin{tabular}{cc}
\hline Property & Virus neutralization titer \\
\hline Antibodies raised against & 3980 \\
BacSC-VP2 & 0 \\
BacSC & 391 \\
CPV & 0 \\
PBS & 0 \\
\hline
\end{tabular}




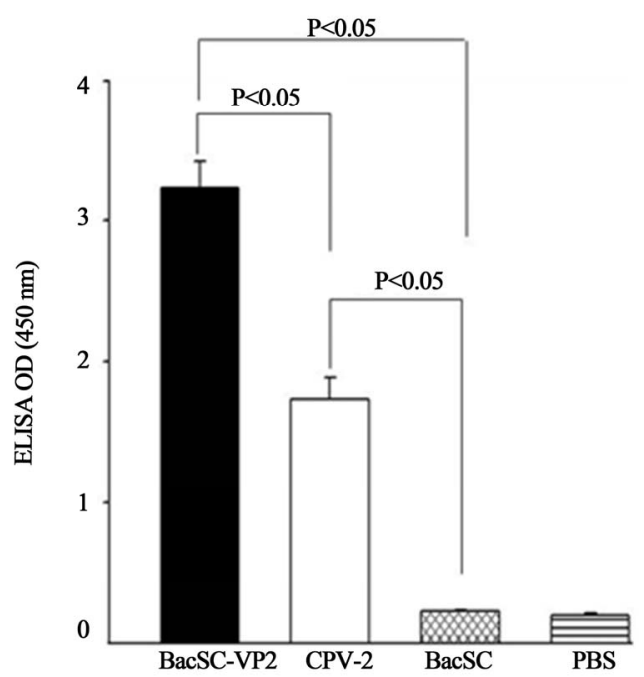

Figure 8. ELISA using serum from BALB/c mice immunized with infected recombinant baculoviruses. Four groups of mice, five female mice in each group, were immunized intraperitioneally with $3 \times 10^{8}$ pfu of purified BacSC-VP2. As negative controls, five mice were injected with purified BacSC $\left(3 \times 10^{8} \mathrm{pfu}\right)$ or phosphate buffered saline (PBS) and five mice were injected with $C P V-2$ vaccine $(1$ dose) as a positive control. Each mouse received one booster shot 2 weeks following initial injection. The blood samples for ELISA were collected 6 weeks after initial injection. The values were mean of three independent experiments. Error bar indicates standard deviation. Comparison with negative controls (BacSC and PBS) were shown to have significant differences $(P<0.05)$.

CPV-2a and $2 \mathrm{~b}$ are distributed worldwide [1,10,16-20]. The new CPV-2c has been detected in many countries [15,21-23]. Several studies demonstrated that VP2-based vaccines can protect or at least increased the survival rate of CPV-2 challenged dogs, as compared to unvaccinated dogs. More recently, Spibey and his colleagues demonstrate that although only a few amino acid substitutions in VP2 that is responsible for its antigenic properties between the original type 2 virus and the CPV $2 a, 2 b$ and 2c $[7,10]$, dogs vaccinated with a CPV-2 live attenuated vaccine can mount a strong immune response and are protected against challenge with the current CPV2c isolates [24].

The recombinant baculoviruses also have a good biosafety profiles, therefore vaccination using recombinant baculoviruses to generate a subunit vaccine should be a safe method. The baculovirus surface display system has become increasingly common as an alternative to the traditional expression systems during the past decade. Recombinant baculoviruses, utilizing the baculovirus gp64 TM and CTD, have been commonly exploited to display various foreign proteins, and have long been used as vaccines in animal models against several viral diseases $[12,13,25-30]$. These proteins can be expressed in insect cell membranes and incorporated into the bacu- lovirus envelope. The expression of the proteins through the baculovirus display system allows rapid production of antigens without the need for purification process. However, none of these studies has looked at the VP2 of CPV-2. The major capsid protein VP2 of CPV-2 plays an important role in the host immune response and virus pathogenicity [6]. In this study, the baculovirus display system has been successfully applied in the production of subunit vaccines for CPV-2 VP2 protein. The recombinant baculovirus BacSC-VP2 expressing histidine-tagged VP2 with the baculovirus gp64 CTD and TM was successfully constructed. After infection, CPV-2 VP2 was expressed and anchored on the plasma membrane of Sf-9 cells and displayed on the viral envelope surface, as demonstrated by confocal microscopy and immunogold electron microscopy. We uncover that the VP2 protein of CPV-2 is translocated to the cell surface. It is worth noting that baculovirus gp64 envelop protein attains it's envelop association only at the time of virion binding and exists as a homotrimer on the viral capsid surface [31]. Although VP2 of CPV-2 and HA of AIV [12] were reported to exist as monomer and homotrimer, respectively, the efficiency of their display on the baculoviral envelope showed no difference $(\mathrm{P}>0.05)$ (Figure 7), suggesting that the protein structure and the presence of the baculovirus gp64 CTD and TM fusions do not alter the growth of the recombinant viruses, efficiency of incorporation, and morphology (BacSC-VP2 and BacSCHA64) and that high titer stocks of these viruses could be achieved.

Animal vaccine tests showed pseudotyped baculovirus BacSC-VP2 elicits significantly higher CPV-2 VP2 antibody titers in mouse models than the negative controls. This demonstrates the feasibility of VP2-pseudotyped baculovirus as a CPV vaccine. Although in this study we used the VP2 protein from the CPV-2 strain, rather than from the recent highly pathogenic CPV-2c. However, dogs vaccinated with a live attenuated $\mathrm{CPV}-2$ vaccine (containing CPV-2 154 strain) can resist challenge with the current CPV2c types [24]. Therefore, the baculovirus/CPV-2 VP2-based vaccine developed in this study holds great promise as a novel platform to prevent the CPV-2 infections. This study opens the way to the development of a recombinant baculovirus vaccine for CPV-2, in which protection is based on the immunogenic properties of the capsid protein.

\section{Acknowledgements}

This work was supported by the grants from Council of Agriculture (101AS-10.2.4 -HI-H2 and 101AS-10.6.1BQ-B6), National Science Council (NSC100-324-B-005009-CC1), and Ministry of Education, Taiwan, under the ATU plan. 


\section{REFERENCES}

[1] U. Truyen, "Evolution of Canine Parvovirus-A Need for New Vaccines?" Veterinary Microbiology, Vol. 117, No. 1, 2006, pp. 9-13. doi:10.1016/j.vetmic.2006.04.003

[2] M. J. Appel, W. Scott and L. E. Carmichael, "Isolation and Immunization Studies of a Canine Parvo-Like Virus from Dogs with Haemorrhagic Enteritis," Veterinary Record, Vol. 105, No. 8, 1979, pp. 156-159. doi:10.1136/vr.105.8.156

[3] W. R. Kelly, "An Enteric Disease of Dogs Reselmbing Feline Panleucopaenia," Austrian Veterinary Journal, Vol. 54, No. 12, 1978, p. 593. doi:10.1111/j.1751-0813.1978.tb02426.x

[4] G. Burtonboy, F. Coignoul and N. Delferriere, "Canine Hemorrhagic Enteritis: Detection of Viral Particles by Electron Microscopy," Archives of Virology, Vol. 61, No. 1-2, 1979, pp. 1-11. doi:10.1007/BF01320586

[5] N. Decaro, C. Desario, M. Campolo, G. Elia, V. Martella, D. Ricci, E. Lorusso and C. Buonavoglia, "Clinical and Virological Findings in Pups Naturally Infected by Canine Parvovirus Type 2 Glu-426 Mutant," Journal of Veterinary Diagnostic Investigation, Vol. 17, No. 2, 2005, pp. 133-138. doi:10.1177/104063870501700206

[6] M. Mochizuki, M. Horiuchi, H. Hiragi, M. C. San Gabriel, N. Yasuda and T. Uno, "Isolation of Canine Parvovirus From a Cat Manifesting Clinical Signs of Feline Panleukopenia," Journal of Clinical Microbiology, Vol. 34, No. 9, 1996, pp. 2101-2105.

[7] W. Yuan and C. R. Parrish, "Comparison of Two Single-Chain Antibodies That Neutralize Canine Parvovirus: Analysis of an Antibody-Combining Site and Mechanisms of Neutralization," Virology, Vol. 269, No. 2, 2000, pp. 471-480. doi:10.1006/viro.2000.0230

[8] M. Nakamura, Y. Tohya, T. Miyazawa, M. Mochizuki, H. T. Phung, N. Nguyen, L. M. Huynh, L. T. Nguyen, P. N. Nguyen, P. V. Nguyen, N. P. Nguyen and H. Akashi, “A Novel Antigenic Variant of Canine Parvovirus from a Vietnamese Dog," Archives of Virology, Vol. 149, No. 11, 2004, pp. 2261-2269. doi:10.1007/s00705-004-0367-y

[9] H. S. Moon, S. A. Lee, S. G. Lee, R. Choi, S. Y. Jeoung, D. Kim and C. Hyun, "Comparison of the Pathogenicity in Three Different Korean Canine Parvovirus 2 (CPV-2) Isolates," Veterinary Microbiology, Vol. 131, No. 1-2, 2008, pp. 47-56. doi:10.1016/j.vetmic.2008.02.016

[10] C. Buonavoglia, V. Martella, A. Pratelli, M. Tempesta, A. Cavalli, D. Buonavoglia, G. Bozzo, G. Elia, N. Decaro and L. Carmichael, "Evidence for Evolution of Canine Parvovirus Type 2 in Italy," Journal of General Virology, 82, Pt. 12, 2001, pp. 3021-3025.

[11] Y. H. Lin, L. H. Lee, W. L. Shih, Y. C. Hu and H. J. Liu, "Baculovirus Surface Display of $\sigma \mathrm{C}$ and $\sigma \mathrm{B}$ Proteins of Avian Reovirus and Immunogenicity of the Displayed Proteins in a Mouse Model," Vaccine, Vol. 26, No. 50, 2008, pp. 6361-6367. doi:10.1016/j.vaccine.2008.09.008

[12] D. G. Yang, Y. C. Chung, Y. K. Lai, H. J. Liu and Y. C. $\mathrm{Hu}$, "Avian Influenza Virus Hemagglutinin Display on Baculovirus Envelope: Cytoplasmic Domain Affects Virus Properties and Vaccine Potential," Molecular Therapy,
Vol.15, No. 5, 2007. pp. 989-996. doi:10.1038/mt.sj.6300131

[13] X. G. Xu, M. T., Chiou, Y. M. Zhang, D. W. Tong, J. H. $\mathrm{Hu}, \mathrm{M}$. T. Zhang and H. J. Liu, "Baculovirus Surface Display of E (rns) Envelope Glycoprotein of Classical Swine Fever Virus," Journal of Virological Methods, Vol. 153, No. 2, 2008, pp. 149-155. doi:10.1016/j.jviromet.2008.07.019

[14] C. R. Parrish, C. F. Aquadro, M. L. Strassheim, J. F. Evermann and J. Y. Sgro, "Mohammed HO. Rapid Antigenic-Type Replacement and DNA Sequence Evolution of Canine Parvovirus," Journal of Virology, Vol. 65, No. 12, 1991, pp. 6544-6552.

[15] N. Decaro, G. Elia, C. Desario, S. Roperto, V. Martella, M. Campolo, A. Lorusso, A. Cavalli and C. Buonavoglia, "A Minor Groove Binder Probe Real-Time PCR Assay for Discrimination between Type 2-Based Vaccines and Field Strains of Canine Parvovirus," Journal of Virological Methods, Vol. 136, No. 1-2, 2006, pp. 65-70. doi:10.1016/j.jviromet.2006.03.030

[16] P. Sagazio, M. Tempesta, D. Buonavoglia, F. Cirone and C. Buonavoglia, "Antigenic Characterization of Canine Parvovirus Strains Isolated in Italy," Journal of Virological Methods, Vol. 73, No. 2, 1998, pp. 197-200. doi:10.1016/S0166-0934(98)00055-X

[17] A. Steinel, E. H. Venter, M. Van Vuuren, C. R. Parrish and U. Truyen, "Antigenic and Genetic Analysis of Canine Parvoviruses in Southern Africa," Onderstepoort Journal of Veterinary Research, Vol. 65, No. 4, 1998, pp. 239-242.

[18] D. Buonavoglia, A. Cavalli, A. Pratelli, V. Martella, G. Greco M. Tempesta and C. Buonavoglia, "Antigenic Analysis of Canine Parvovirus Strains Isolated in Italy," New Microbiology, Vol. 23, No. 1, 2000, pp. 93-96.

[19] U. Truyen, A. Steinel, L. Bruckner, H. Lutz and K. Mostl, "Distribution of Antigen Types of Canine Parvovirus in Switzerland, Austria and Germany," Schweiz Arch Tierheilkd, Vol. 142, No. 3, 2000, pp. 115-119.

[20] V. Martella, A. Cavalli, A. Pratelli, G. Bozzo, M. Camero, D. Buonavoglia, D. Narcisi, M. Tempesta and C. Buonavoglia, "A Canine Parvovirus Mutant Is Spreading in Italy," Journal of Clinical Microbiology, Vol. 42, No. 3, 2004, pp. 1333-1336. doi:10.1128/JCM.42.3.1333-1336.2004

[21] N. Decaro, C. Desario, D. D. Addie, V. Martella, M. J. Vieira, G. Elia, A. Zicola, C. Davis, G. Thompson, E. Thiry, U. Truyen and C. Buonavoglia, "The Study Molecular Epidemiology of Canine Parvovirus", Emerging Infectous Diseases, Vol. 13, No. 8, 2007, pp. 1222-1224. doi:10.3201/eid1308.070505

[22] C. Hong, N. Decaro, C. Desario, P. Tanner, M. C. Pardo, S. Sanchez, C. Buonavoglia and J. T. Saliki, "Occurrence of Canine Parvovirus Type 2c in the United States," Journal of Veterinary Diagnostic Investigation, Vol. 19, No. 5, 2007, pp. 535-539. doi: $10.1177 / 104063870701900512$

[23] R. Perez, L. Francia, V. Romero, L. Maya, I. Lopez and M. Hernandez, "First Detection of Canine Parvovirus Type 2c in South America," Veterinary Microbiology, 
Vol. 124, No. 1-2, 2007, pp. 147-152. doi:10.1016/j.vetmic.2007.04.028

[24] N. Spibey, N. M. Greenwood, D. Sutton, W. S. K. Chalmers and I. Tarpey, "Canine Parvovirus Type 2 Vaccine Protects Against Virulent Challenge with Type 2c Virus," Veterinary Microbiology, Vol. 128, No. 1-2, 2008, pp. 48-55. doi:10.1016/j.vetmic.2007.09.015

[25] D. Mottershead, I. van der Linden, C. H. von Bonsdorff, K. K. Keinanen and C. Oker-Blom, "Baculoviral Display of the Green Fluorescent Protein and Rubella Virus Envelope Proteins," Biochemical and Biophysical Research Communications, Vol. 238, No. 3, 1997, pp. 717-722. doi:10.1006/bbrc. 1997.7372

[26] M. M. Rahman, M. S. Shalia and K. P. Gopinathan, "Baculovirus Display of Fusion Protein of Peste des Petits Ruminants Virus and Hemagglutination Protein of Rinderpest Virus and Immunogenicity of the Displayed Proteins in Mouse Model," Virology, Vol. 317, No. 1, 2003, pp. 36-49. doi:10.1016/j.virol.2003.08.022

[27] X. G. Xu and H. J. Liu, "Baculovirus Surface Display of E2 Envelope Glycoprotein of Classical Swine Fever Virus and Immunogenicity of the Displayed Proteins in a Mouse Model," Vaccine, Vol. 26, No. 43, 2008, pp. 54555460 .
[28] X. G. Xu, D. W. Tong, M. T. Chiou, Y. C. Hsieh, W. L. Shih, C. D. Chang, M. H. Liao, Y. M. Zhang and H. J. Liu, "Baculovirus Surface Display of NS3 Nonstructural Protein of Classical Swine Fever Virus," Journal of Virological Methods, Vol.159, No. 2, 2009, pp. 259-264. doi:10.1016/i.jviromet.2009.04.013

[29] X. G. Xu, Z. S. Wang, G. Zhang, Z. C. Li, H. N. Zhao, W. Li, D. W. Tong and H. J. Liu, "Baculovirus Surface Display of E Envelope Glycoprotein of Japanese Encephalitis Virus and Its Immunogenicity of the Displayed Proteins in Mouse and Swine Models," Vaccine, Vol. 29, No. 4, 2011, pp. 636-643. doi:10.1016/j.vaccine.2010.11.045

[30] X. G. Xu, Z. S. Wang, G. Zhang, Z. C. Li, H. N. Zhao, W. Li, D. W. Tong and H. J. Liu, "Baculovirus Virions Displaying Infectious Bursal Disease Virus VP2 Protein Protect Chickens against Infectious Bursal Disease Virus Infection," Avian Diseases, Vol. 55, No. 2, 2011, pp. 223 229. doi:10.1637/9597-111210-Reg.1

[31] A. G. Oomens, S. A. Monsma and G. W. Blissard, "The Baculovirus GP64 Envelope Fusion Protein: Synthesis, Oligomerization and Processing," Virology, Vol. 209, No. 2, 1995, pp. 592-603. doi:10.1006/viro.1995.1291 